\title{
CARACTERÍSTICAS DEL MALTRATO HACIA ESTUDIANTES DE MEDICINA DE UNA UNIVERSIDAD PÚBLICA DEL PERÚ
}

\author{
Fernando Munayco-Guillén 1,2,a Anais Cámara-Reyes,3,a, L. Jaime Muñoz-Tafur,a, Hugo Arroyo-Hernández \\ Christian R. Mejia ${ }^{5, b}$, Felix Lem-Arce ${ }^{3, c}$, Ubaldo E Miranda-Soberón ${ }^{3,6, d}$
}

\begin{abstract}
RESUMEN
Objetivos. Determinar las características del maltrato hacia estudiantes de Medicina de una universidad pública en la provincia de Ica, Perú. Materiales y métodos. Investigación transversal realizada el 2012 en una muestra probabilística de estudiantes de Medicina de primer a sexto año de la Universidad Nacional San Luis Gonzaga. Se elaboró una encuesta con un total de 23 ítems en una escala likert, para medir la percepción de maltrato de tipo psicológico, físico, académico y sexual; se usaron análisis estadísticos univariados y bivariados. Resultados. Se encuestaron 281 estudiantes. La percepción de maltrato psicológico fue $96,8 \%$, académico $86,8 \%$, físico $62,6 \%$ y sexual $20,6 \%$; el maltrato físico se incrementó durante el ciclo de estudio clínico-quirúrgico $(p=0,001)$. Los médicos docentes y médicos residentes fueron los principales agresores. Estudiantes hombres reportaron con mayor frecuencia haber recibido tareas como castigo, no recibir los créditos por su trabajo, maltrato físico, amenazas verbales, insultos o recibir burlas con respecto a su etnia; mientras que el maltrato sexual fue mayor en mujeres. El reporte de maltrato sexual fue más frecuente en la universidad $(45,3 \%, p=0,002)$ y el hospital $(45,0 \%, p=0,046)$. Las mujeres reportaron con mayor frecuencia no saber a quién o dónde acudir para denunciar el maltrato $(54,6 \%, p=0,042)$ y no denunciarlo porque se detuvo el maltrato $(56,9 \%, p=0,048)$. Conclusiones. Existe una alta prevalencia de maltrato, donde las características de los estudiantes según el sexo, los ciclos de estudio y el agresor permiten identificar los tipos de maltrato que reciben los estudiantes de medicina.
\end{abstract}

Palabras clave: Estudiantes de medicina; Maltrato; Escuelas médicas (fuente: DeCS BIREME)

\section{CHARACTERISTICS OF MISTREATMENT IN MEDICAL STUDENTS OF A PUBLIC UNIVERSITY IN PERU}

\begin{abstract}
Objectives. To determine the characteristics of mistreatment among medical students at a public university in the province Ica, Peru. Materials and methods. This cross-sectional study was performed in 2012, and used a probability sample of medical students (freshman to sixth year) at the Universidad Nacional San Luis Gonzaga. A 23-item survey with a Likert scale was used to measure the students' perceptions of psychological, physical, academic and sexual mistreatment. Univariate and bivariate statistical analysis were performed. Results. A total of 281 students were surveyed. The perception of psychological mistreatment was $96.8 \%$; academic, $86.8 \%$; physical, $62.6 \%$ and sexual, $20.6 \%$. Physical abuse increased during the clinical-surgical study cicle $(p=0.001)$. Medical doctors and residents were the main aggressors. Male students reported of receiving assignments as a punishment, not receiving credits for their work, physical mistreatment, verbal threats, insults, or being teased because of their etnic background. Female students were more likely to have experienced sexual abuse. Report of sexual abuse frequently ocurred at the university $(45.3 \%, p=0.002)$ and hospital $(45.0 \%, p=0.046)$. Women frequently reported not knowing to whom or where to denounce the abuse $(54.6 \%, p=0.042)$ and not reporting it because it stopped $(56.9 \%, p=0.048)$. Conclusions. There is a high prevalence of abuse among students in which their characteristics as sex, level of study and aggressor allow to identify the types of abuse that they receive.
\end{abstract}

Keys words: Students, medical; Mistreatment; Schools, medical (source: MeSH NLM).

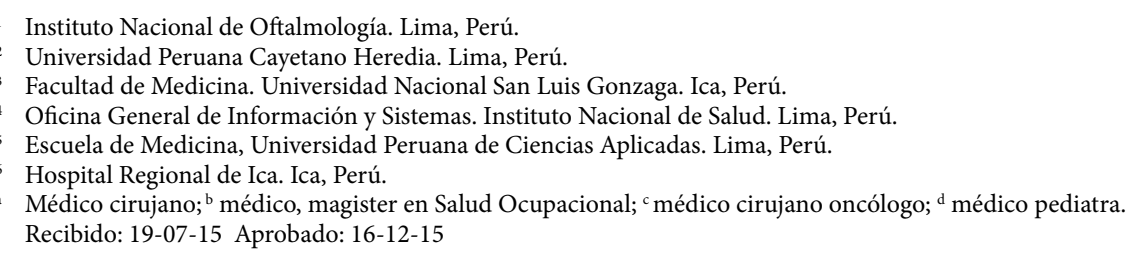




\section{INTRODUCCIÓN}

La formación de los recursos humanos en salud se distingue de otras carreras profesionales, por ser muy jerarquizada, siendo el estudiante de Medicina vulnerable a recibir algún tipo de maltrato por parte de un superior ${ }^{(1,2)}$. Los individuos que pueden actuar como agresores, por lo general, son los médicos docentes, médicos residentes, pacientes, trabajadores del hospital, trabajadores de la universidad y otros estudiantes de Medicina ${ }^{(3)}$. Una revisión sobre el maltrato hacia estudiantes de Medicina muestra que es un problema con altas prevalencias y que no ha disminuido en el tiempo ${ }^{(4)}$

Los estudiantes de Medicina que son víctimas de maltrato desde etapas tempranas de su formación, podrían percibir al maltrato como un hecho normal e, incluso, útil como experiencia de aprendizaje, justificando y perpetuando el proceder del maltrato en la enseñanza. Tradicionalmente el maltrato se ha considerado como parte de la misma formación del médico, mientras que los estudiantes de otras profesiones no tienen esa percepción, por lo tanto, tienen mayor disposición a reportar incidentes de maltrato ${ }^{(5-8)}$.

Cualquier forma de maltrato podría afectar la autoestima y el comportamiento moral o social del estudiante, además de su futuro desempeño profesional, por ello es necesario identificarlo tempranamente para prevenir sus consecuencias ${ }^{(4,5)}$. El gran número de informes sobre el tema indican la necesidad de una revisión a las políticas existentes sobre acoso, discriminación y maltrato en las facultades de Medicina ${ }^{(6)}$. El maltrato hacia estudiantes es un tema altamente sensible y poco estudiado en Sudamérica, en especial en Perú, donde por tradición se conoce que este mal proceder ha estado siempre vinculada al ambiente de la formación médica ${ }^{(3,4)}$. El presente estudio tiene como objetivo determinar las características del maltrato percibido por los estudiantes de Medicina de una universidad pública de una provincia del Perú.

\section{MATERIALES Y MÉTODOS}

\section{DISEÑO Y POBLACIÓN DE ESTUDIO}

Se realizó una investigación de tipo observacional, transversal analítico, durante mayo y junio de 2012, en la Facultad de Medicina de la Universidad Nacional San Luis Gonzaga, en la provincia de Ica en la costa sur del Perú.

La selección de los participantes se realizó mediante un muestreo aleatorio estratificado para cada uno de los ciclos de estudios en la universidad correspondientes a los seis años de estudios, calculando una muestra significativa con un nivel de confianza del $95 \%$ por cada ciclo de estudios, el tamaño de la muestra fue calculada mediante el programa Epi Info versión 3.0.

Los criterios de inclusión fueron: estar matriculado y desear participar del estudio. No se incluyeron a los estudiantes del primer ciclo debido a que el tiempo que llevan estudiando es muy corto, por lo que la percepción de maltrato puede estar sujeta a sesgo de concepto o de memoria. Tampoco se incluyeron a los estudiantes de internado por tener una situación educativa distinta a los demás estudiantes fuera de las aulas de estudio.

\section{TIPOS DE MALTRATO}

Los principales tipos de maltrato fueron seleccionados según las directrices para afrontar la violencia laboral en el sector salud de la Organización Internacional del Trabajo, las preguntas que fueron incluidas en la encuesta para cada uno de estos tipos de maltrato fueron seleccionadas de estudios similares publicados. Los tipos de maltrato estudiados fueron: psicológico, físico, académico y sexual, cuyas definiciones son las siguientes.

Maltrato psicológico. Es el uso premeditado del poder, o amenazas verbales, que puede dañar la esfera mental, espiritual, moral o social. Comprende el abuso verbal (insultos, apodos, gritos), la intimidación, el atropello, el acoso y las amenazas, burlas, la humillación en público, el menosprecio, la discriminación en todas sus formas (de género, religioso, racial, socioeconómica, por la edad, o por rendimiento académico) ${ }^{(21,26)}$.

Maltrato físico. Es el empleo de la fuerza física contra otra persona o grupo, que produce daños físicos, sexuales o psicológicos, incluyendo en ellas las palizas, patadas, bofetadas, empujones, pellizcos, lanzar a otra persona un objeto, asignación de trabajos excesivos, o exposición a procedimientos sin la protección adecuada ${ }^{(26,27)}$.

Maltrato académico. Son conductas inadecuadas por parte del docente hacia el alumno, en el contexto del proceso enseñanza-aprendizaje, que puede afectar su desempeño y su evaluación ${ }^{(14,23)}$.

Maltrato sexual. Es una conducta de carácter sexual, no correspondida ni deseada, que resulta ofensiva para la otra persona y es causa de que esta persona se sienta amenazada, humillada, o avergonzada. Se consideró como maltrato sexual a las insinuaciones verbales sexuales o comentarios obscenos, lenguaje corporal ofensivo de tipo sexual, discriminación de género, propuestas indecentes, tocamientos indebidos, discriminación de preferencia sexual y el chantaje sexual ${ }^{(10,26)}$. 


\section{INSTRUMENTO}

Se elaboró una encuesta titulada: Cuestionario de Percepción de Maltrato en el Estudiante de Medicina, diseñado por los autores y basado en la revisión de publicaciones realizadas en el tema, para establecer una definición conceptual del maltrato y sus diferentes formas en estudiantes de medicina. El instrumento fue sometido a una prueba piloto en 80 estudiantes de diversos años, seleccionados de manera aleatoria, obteniendo un alfa de Cronbach de 0,8 . Los resultados de la validación del instrumento se presentan en el anexo 1,2 y 3 .

El instrumento elaborado tuvo tres secciones: 1). Características sociodemográficas y educativas; 2) Percepción del maltrato según los cuatro componentes evaluados y que contenía un total de 23 ítems siendo: a) maltrato psicológico (si le gritaron, recibido comentarios negativos/despectivos, humillado, insultado, recibido críticas injustificadas, recibido amenazas verbales y burlas por el género o la etnia); b) maltrato físico (si lo golpearon, expuesto a riesgos innecesarios o asignado trabajos excesivos); c) maltrato académico (asignado tareas como castigo, amenazado con desaprobar, experimentado competencia desleal $\mathrm{u}$ otros han tomado el crédito por su trabajo) y d) maltrato sexual (tuvieron discriminación por el género, hecho insinuaciones verbales sexuales/comentarios obscenos, discriminado por mi preferencia sexual, hecho propuestas indecentes, mostrado imágenes ofensivas de tipo sexual, tocado sin su consentimiento, mostrado un lenguaje corporal ofensivo de tipo sexual o chantajeado sexualmente). 3 ), Lugar donde se había realizado el maltrato; persona que había realizado el maltrato; denuncia del maltrato, y motivos para no denunciar el maltrato.

\section{PROCEDIMIENTOS}

La recolección de los datos fue realizada por los autores, de la siguiente manera: se obtuvo la lista oficial de estudiantes matriculados del ciclo 2 al 12 de la Secretaría Académica de la universidad, consignándose un total de 492 estudiantes matriculados en el año académico 2012. Se coordinó con el representante estudiantil de cada ciclo para poder realizar el estudio cuando estén presentes todos los estudiantes. Al llegar al salón de clases se explicó el motivo, los objetivos y la importancia del estudio. Se procedió a llamar de manera aleatoria a los integrantes del ciclo hasta completar el número de muestra requerida por ciclo. A cada estudiante seleccionado se le entregó el consentimiento informado y se solicitó su firma como aceptación para participar en el estudio; se procedió a recoger las hojas de consentimiento informado y luego se entregó el cuestionario sin consignar la identificación del participante. Se aplicó el cuestionario sin límite de tiempo, y al finalizarlo se le pidió a cada estudiante que lo doblara y lo depositara en un ánfora.

\section{ANÁLISIS ESTADÍSTICO}

Cada una de las subcategorías de maltrato fueron respondidas mediante una escala likert de 5 alternativas (desde nunca=1 hasta siempre=5). Para motivos de análisis se categorizó cada subcategoría, luego se realizaron cálculos de frecuencias y análisis univariados, se utilizaron las pruebas de chi cuadrado, test exacto de Fisher y $t$ de student, según sea el caso, utilizando el programa estadístico STATA 12.

\section{ASPECTOS ÉTICOS}

El protocolo del estudio fue revisado y aprobado por el Comité de Ética del Hospital Regional de Ica. La participación de los estudiantes fue voluntaria previo consentimiento informado y firma de participación, el llenado de la encuesta fue anónimo, asegurando en todo momento la confidencialidad de los datos.

\section{RESULTADOS}

La población encuestada estuvo conformada por 281 estudiantes de Medicina del primer al sexto año. La media para la edad fue de $22 \pm 3$ años (rango de 16 a 35$)$; el $50,5 \%$ (142) fueron hombres, el $17,1 \%$ (48) procedían de alguna región de la sierra o la selva del Perú, el 53,7\% (151) se encontraba cursando los ciclos de ciencias básicas correspondientes a los tres primeros años de estudios, el 19,6\% (55) había repetido algún curso en el ciclo de estudio anterior.

El tipo de maltrato más frecuente, según el componente psicológico, fue haber recibido más de alguna vez comentarios negativos o despectivos con $89,8 \%$, y haber recibido críticas injustificadas con $82,1 \%$; según el componente académico, el haber experimentado una competencia desleal con $74,0 \%$ (208); según el componente físico, haber sido asignado con trabajos excesivos con $56,6 \%$ (159), y según el componente sexual, haber recibido insinuaciones verbales sexuales o comentarios obscenos con $20,6 \%$ (58) o haber percibido un lenguaje corporal ofensivo de tipo sexual (gestos obscenos, miradas, roses, tocamientos o acercamientos innecesarios) $17,1 \%$ (48) Figura 1.

La Tabla 1 muestra los ocho ítems que presentaron diferencias significativas en la percepción de maltrato según el sexo de los estudiantes, de los cuales dos fueron del componente sexual, dos del componente académico, uno del componente físico y tres del componente psicológico. 


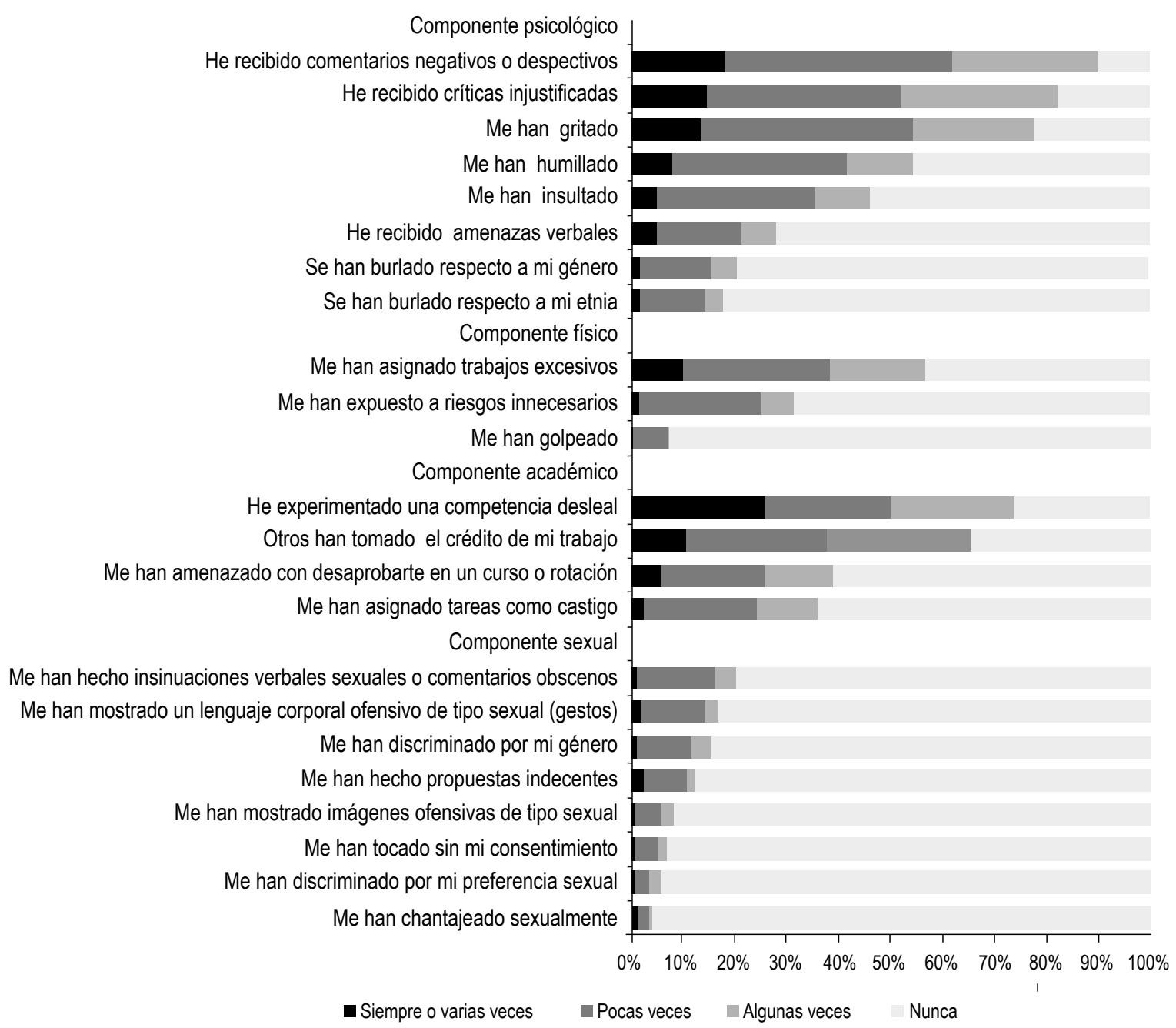

Figura 1. Percepción de maltrato en estudiantes de Medicina de una universidad pública de una provincia en Perú, 2012

Tabla 1. Diferencias halladas en la percepción de maltrato según sexo en estudiantes de Medicina de una universidad pública de una provincia en Perú, 2012

\begin{tabular}{|c|c|c|c|c|c|}
\hline \multirow{2}{*}{ Tipo de maltrato } & \multicolumn{2}{|c|}{ Hombre } & \multicolumn{2}{|c|}{ Mujer } & \multirow{2}{*}{ Valor $p$} \\
\hline & $\mathbf{n}$ & $\%$ & $\mathbf{n}$ & $\%$ & \\
\hline Se han burlado respecto a mi etnia * & 33 & 23,2 & 17 & 12,2 & 0,016 \\
\hline He recibido amenazas verbales * & 53 & 37,3 & 25 & 18,0 & 0,001 \\
\hline Me han insultado * & 85 & 59,9 & 44 & 31,7 & 0,001 \\
\hline Me han golpeado ** & 18 & 12,7 & 3 & 2,2 & 0,001 \\
\hline Me han asignado tareas como castigo*** & 61 & 43,0 & 41 & 29,5 & 0,019 \\
\hline Otros han tomado el crédito de mi trabajo*** & 105 & 73,9 & 80 & 57,5 & 0,004 \\
\hline Me han discriminado por mi género**** & 15 & 10,6 & 29 & 20,9 & 0,018 \\
\hline Me han hecho insinuaciones verbales sexuales o comentarios obscenos ${ }^{* * * *}$ & 21 & 14,8 & 37 & 26,6 & 0,014 \\
\hline
\end{tabular}

*Maltrato psicológico, ${ }^{* *}$ maltrato físico, ${ }^{* * *}$ maltrato académico, ${ }^{* * * *}$ maltrato sexual 
La agresión recibida de los médicos docentes fue superior en los componentes psicológico $99,4 \%$ $(p=0,006)$; académico $91,3 \%(p=0,010)$; físico $68,5 \%$ $(p=0,001)$, y sexual $42,7 \%(p=0,004)$, y de los médicos residentes en los componentes académico $100 \%$ $(p=0,034)$; físico $82,1 \% \quad(p=0,025)$, y sexual $64,3 \%$ $(p=0,002)$; el maltrato por parte de otros estudiantes fue mayor para el componente académico $96,2 \%(p=0,004)$. El lugar donde se percibió algún tipo de maltrato fue más frecuente para la universidad en los tipos psicológico $99,4 \%(p=0,006)$; académico $91,3 \%(p=0,010)$, y sexual $45,3 \%(p=0,002)$; y dentro del hospital el maltrato más frecuente fue de tipo sexual $45,0 \%(p=0,046)$; haber recibido algún tipo de maltrato físico fue mayor en estudiantes que cursan estudios en las áreas clínico- quirúrgicas y migrantes $77,1 \% \quad(p=0,023)$, siendo, además, la media de la edad $(23,1 \pm 2,68)$ superior a la de quienes no reportaron algún tipo de maltrato físico $(p=0,001)$ Tabla 2.

En la Tabla 3 se muestran los resultados de la percepción de maltrato según el ciclo académico y el agresor, siendo el médico docente y el médico residente los principales agresores durante el ciclo clínico-quirúrgico.

De acuerdo con los motivos para no reportar el maltrato, las mujeres presentaron frecuencias superiores para "no saber a quién o a dónde acudir para denunciar el maltrato" $54,6 \%(p=0,042)$ y "no denuncié porque se detuvo el maltrato" 56,9\% ( $p=0,048)$ Tabla 4.

Tabla 2. Tipo de maltrato según características del agresor y de los estudiantes de Medicina de una universidad pública de una provincia en Perú

\begin{tabular}{|c|c|c|c|c|c|c|c|c|c|c|c|c|}
\hline & \multicolumn{3}{|c|}{ Psicológico } & \multicolumn{3}{|c|}{ Académico } & \multicolumn{3}{|c|}{ Físico } & \multicolumn{3}{|c|}{ Sexual } \\
\hline & $\mathbf{n}$ & $\%$ & $\mathbf{p}^{*}$ & $\mathbf{n}$ & $\%$ & $\mathbf{p}$ & $\mathbf{n}$ & $\%$ & $\mathbf{p}$ & $\mathbf{n}$ & $\%$ & $\mathbf{p}$ \\
\hline Edad media (DE) & 272 & $21,9(3,03)$ & 0,135 & 244 & $21,7(3,02)$ & 0,325 & 176 & $23,1(2,68)$ & 0,001 & 106 & $22,1(3,03)$ & 0,297 \\
\hline \multicolumn{13}{|l|}{ Sexo } \\
\hline Hombre & 137 & 96,5 & 1,000 & 124 & 87,3 & 0,806 & 89 & 62,7 & 0,988 & 47 & 33,1 & 0,106 \\
\hline Mujer & 135 & 97,1 & & 120 & 86,3 & & 87 & 62,6 & & 59 & 42,5 & \\
\hline \multicolumn{13}{|l|}{ Ciclo de estudio } \\
\hline Ciencias básicas & 145 & 96,0 & 0,512 & 136 & 90,1 & 0,084 & 48 & 31,8 & 0,001 & 53 & 35,1 & 0,328 \\
\hline Clínico quirúrgico & 127 & 97,7 & & 108 & 83,1 & & 128 & 98,5 & & 53 & 40,8 & \\
\hline \multicolumn{13}{|l|}{ Repitió algún curso } \\
\hline No & 218 & 96,5 & 0,446 & 198 & 87,6 & 0,434 & 137 & 60,6 & 0,157 & 80 & 35,4 & 0,103 \\
\hline Sí & 54 & 98,2 & & 46 & 83,6 & & 39 & 70,9 & & 26 & 47,3 & \\
\hline \multicolumn{13}{|l|}{ Procedencia } \\
\hline Costa & 225 & 96,6 & 1,000 & 201 & 86,3 & 0,536 & 139 & 59,7 & 0,023 & 91 & 39,1 & 0,310 \\
\hline Sierra o selva & 47 & 97,9 & & 43 & 89,6 & & 37 & 77,1 & & 15 & 31,3 & \\
\hline \multicolumn{13}{|c|}{ Agresor médico docente } \\
\hline Nunca & 67 & 89,3 & 0,001 & 56 & 74,7 & 0,001 & 35 & 46,7 & 0,001 & 18 & 24,0 & 0,004 \\
\hline Alguna vez & 205 & 99,5 & & 188 & 91,3 & & 141 & 68,5 & & 88 & 42,7 & \\
\hline \multicolumn{13}{|c|}{ Agresor médico residente } \\
\hline Nunca & 244 & 96,4 & 0,606 & 216 & 85,4 & $0,034^{*}$ & 153 & 60,5 & 0,025 & 88 & 34,8 & 0,002 \\
\hline Alguna vez & 28 & 100 & & 28 & 100 & & 23 & 82,1 & & 18 & 64,3 & \\
\hline \multicolumn{13}{|l|}{ Agresor estudiante } \\
\hline Nunca & 195 & 96,1 & 0,452 & 169 & 83,3 & 0,004 & 128 & 63,1 & 0,814 & 71 & 35,0 & 0,125 \\
\hline Alguna vez & 77 & 98,7 & & 75 & 96,2 & & 48 & 61,5 & & 35 & 44,9 & \\
\hline \multicolumn{13}{|c|}{ Maltrato en la universidad } \\
\hline Nunca & 112 & 93,3 & 0,006 & 97 & 80,8 & 0,010 & 74 & 61,7 & 0,772 & 33 & 27,5 & 0,002 \\
\hline Alguna vez & 160 & 99,4 & & 147 & 91,3 & & 102 & 63,4 & & 73 & 45,3 & \\
\hline \multicolumn{13}{|c|}{ Maltrato en el hospital } \\
\hline Nunca & 164 & 95,4 & 0,160 & 145 & 84,3 & 0,115 & 100 & 58,1 & 0,050 & 57 & 33,1 & 0,046 \\
\hline Alguna vez & 108 & 99,1 & & 99 & 90,8 & & 76 & 69,7 & & 49 & 45,0 & \\
\hline
\end{tabular}

* Prueba exacta de Fisher DE: desviación estándar 
Tabla 3. Tipo de maltrato según agresor en estudiantes de Medicina de ciencias básicas y clínico-quirúrgicas

\begin{tabular}{|c|c|c|c|c|c|}
\hline \multirow{2}{*}{ Tipo de maltrato según agresor } & \multicolumn{2}{|c|}{ Ciencias básicas } & \multicolumn{2}{|c|}{ Clínico-quirúrgico } & \multirow{2}{*}{ Valor $\mathrm{p}$} \\
\hline & $\mathbf{n}$ & $\%$ & $\mathbf{n}$ & $\%$ & \\
\hline \multicolumn{6}{|l|}{ Médico docente } \\
\hline Psicológico & 98 & 67,6 & 107 & 84,3 & 0,001 \\
\hline Académico & 94 & 69,1 & 94 & 87,0 & 0,001 \\
\hline Físico & 36 & 75,0 & 105 & 82,0 & 0,298 \\
\hline Sexual & 40 & 75,5 & 48 & 90,6 & 0,038 \\
\hline \multicolumn{6}{|l|}{ Estudiante } \\
\hline Psicológico & 47 & 32,4 & 30 & 23,6 & 0,108 \\
\hline Académico & 46 & 33,8 & 29 & 26,9 & 0,241 \\
\hline Físico & 18 & 37,5 & 30 & 23,4 & 0,062 \\
\hline Sexual & 21 & 39,6 & 14 & 26,4 & 0,148 \\
\hline \multicolumn{6}{|l|}{ Médico residente } \\
\hline Psicológico & 5 & 3,5 & 23 & 18,1 & 0,001 \\
\hline Académico & 5 & 3,7 & 23 & 21,3 & 0,001 \\
\hline Físico & 0 & 0,0 & 23 & 18,0 & 0,002 \\
\hline Sexual & 3 & 5,7 & 15 & 28,3 & 0,002 \\
\hline
\end{tabular}

Tabla 4. Motivos para no denunciar el maltrato según el sexo de los estudiantes de Medicina

\begin{tabular}{|c|c|c|c|c|c|c|}
\hline \multirow{2}{*}{ Si usted experimentó maltrato y no lo denunció, ¿cuál fue el motivo? } & \multirow[b]{2}{*}{ Total } & \multicolumn{2}{|c|}{ Hombre } & \multicolumn{2}{|c|}{ Mujer } & \multirow[b]{2}{*}{ Valor $p$} \\
\hline & & $\mathbf{n}$ & $\%$ & $\mathbf{n}$ & $\%$ & \\
\hline Pensar que la denuncia del maltrato quedará en nada & 125 & 58 & 40,9 & 67 & 48,2 & 0,215 \\
\hline Considerar que denunciar el maltrato me traería problemas & 157 & 75 & 53,2 & 82 & 59,0 & 0,328 \\
\hline Pensar que la denuncia influiría negativamente en mis calificaciones & 182 & 87 & 61,3 & 95 & 68,4 & 0,214 \\
\hline No era tan importante como para ser denunciada a las autoridades & 180 & 87 & 61,3 & 93 & 67,4 & 0,285 \\
\hline No consideré que el problema sería tratado con justicia & 152 & 74 & 52,1 & 78 & 56,1 & 0,501 \\
\hline No sabía a quién o a dónde acudir para denunciar el maltrato & 165 & 75 & 52,8 & 90 & 64,8 & 0,042 \\
\hline Tenía miedo que mi denuncia no sería confidencial & 109 & 57 & 40,1 & 52 & 37,4 & 0,639 \\
\hline Tenía miedo de que no creyeran mi testimonio & 145 & 73 & 51,8 & 72 & 51,8 & 0,997 \\
\hline $\begin{array}{l}\text { No reconocer la experiencia vivida como maltrato en el momento en el que ocu- } \\
\text { rrió }\end{array}$ & 154 & 76 & 53,5 & 78 & 56,5 & 0,614 \\
\hline Tenía miedo de denunciar el maltrato & 117 & 57 & 40,1 & 60 & 43,2 & 0,607 \\
\hline Podría influir negativamente en mi carrera profesional en el futuro & 128 & 65 & 45,8 & 63 & 45,3 & 0,940 \\
\hline Tenía vergüenza y no quería ser señalado por los demás & 124 & 58 & 40,9 & 66 & 47,5 & 0,263 \\
\hline No denuncié porque se detuvo el maltrato & 109 & 47 & 33,1 & 62 & 44,6 & 0,048 \\
\hline No quería pensar más en el maltrato sufrido & 124 & 63 & 44,4 & 61 & 43,9 & 0,935 \\
\hline
\end{tabular}

\section{DISCUSIÓN}

El presente estudio ha permitido determinar cuáles son las características del maltrato hacia los estudiantes de Medicina, y los motivos por los que el maltrato no fue denunciado. Los hallazgos mostraron una alta prevalencia para el maltrato de tipo psicológico seguido del académico, el físico y el sexual, con frecuencias que varian según el agresor, el ciclo de estudios y el sexo de los estudiantes. 
El uso por parte de algunos médicos docentes, de acciones equivocadas por reforzar el aprendizaje mediante comentarios negativos, gritos, amenazas o críticas injustificadas, son las formas de maltrato psicológico más frecuente. La persistencia de este tipo de maltrato podría fomentar comportamientos insensibles y punitivos que se transmiten por los que, a su vez, han sido maltratados, el resultado no deseado se agrava cuando estas conductas son dirigidas a pacientes y colegas ${ }^{(9)}$. El alto reporte de maltrato psicológico se mantiene tanto en los primeros tres años de estudios, o ciclo de ciencias básicas, asi como en el ciclo clínico-quirúrgico, donde los estudiantes interactúan mas tiempo en ambientes hospitalarios, estos resultados son similares al reportado por otros autores con valores del $98 \%$ en estudiantes de Medicina de México y Colombia ${ }^{(10,11)}$, y superior al reportado en países como Chile o Nigeria con el 79,9 y $87,4 \%$ respectivamente ${ }^{(12,13)}$.

El maltrato académico por parte de algunos médicos docentes y residentes se incrementó durante el ciclo clínicoquirúrgico, probablemente debido a la mayor interacción con ellos y donde las amenaza con desaprobar un curso son su principal forma. Si bien el reporte de maltrato académico por otro estudiante fue significativamente mayor en la evaluación a todos los estudiantes, no presentó diferencias al compararlo entre ciclos de estudio.

Si bien no existen diferencias entre el sexo de los estudiantes y los tipos de maltrato, algunos de los items de maltrato académico, físico y psicológico fueron mayores en hombres, mientras que en otras publicaciones, el maltrato sexual fue mayor en mujeres ${ }^{(14-16)}$. Estos resultados deben ponerse en el contexto sociocultural de cada región o país. Si bien existe un incremento de mujeres estudiantes de Medicina en las universidades, los reportes de discriminación de género y el acoso sexual son comunes ${ }^{(17,18)}$.

En la evaluación de los motivos para no denunciar el maltrato, la mayoría de mujeres manifestaron no saber donde acudir para denunciarlo, o no hacerlo porque el maltrato se detuvo; esto pone de manifiesto que siendo el maltrato un fenómeno percibido desde etapas universitarias tempranas, es negativo que no existan los medios necesarios para denunciarlo, ni tener que sentir un impacto emocional por ello. Al respecto, los responsables de la educación en instituciones médicas deben controlar la discriminación de género y la prevención del acoso sexual.

Una explicación desde la psicología social por la cual los estudiantes no denuncian estas conductas abusivas, aceptando el abuso como una herramienta pedagógica, se debería a un fenómeno de intercambio de capitales definido por Bourdieu como todo aquello que tenga valor simbólico en una sociedad determinada ${ }^{(19)}$. El estudiante al ingresar a una comunidad médica u hospitalaria con normas y códigos propios, debe adaptarse para poder formar parte de esta, una vez adaptado a este ambiente, como sucede con los estudiantes de últimos años, muchos de los comportamientos abusivos son considerados como manifestaciones comunes en la interacción institucional.

Al igual que otros reportes que muestran altas frecuencias de discriminación en minorías étnicas ${ }^{(20,21)}$, en el presente estudio se encontró un mayor reporte de maltrato físico en estudiantes procedentes de la sierra o selva lo cual estaría relacionado con la condición de vulnerabilidad de este grupo de estudiantes, como el hecho de vivir solos, no tener cerca el soporte familiar, así como al enfrentarse a nuevos ambientes y comportamientos culturales que podrían ser hostiles y los haría más propensos a sufrir abusos de sus compañeros o docentes; por ello es importante considerar a este grupo de migrantes en futuros estudios.

El ciclo de estudio en áreas clínico-quirúrgicas, incide en el incremento del maltrato físico y el reporte de maltrato por parte de los médicos docentes y residentes. Estos hallazgo son similares al de otras investigaciones, donde el maltrato se incrementa al avanzar la carrera e interactuar en ambientes hospitalarios, debido a que el número de estudiantes por docente es menor y el contacto directo es casi permanente, lo que permite que la agresión psicológica o académica se perciba con mayor frecuencia ${ }^{(22-25)}$.

Al igual que otros países el reporte de maltrato hacia los estudiantes de Medicina es un problema que ha mostrado alta prevalencia, por lo que en la actualidad ya existen medidas como la de la Comisión de Enlace para la Educación Médica en EE. UU. que exige la evaluación del maltrato en los estándares de acreditación de las facultades de Medicina. Estrategias para reducir este problema requieren el uso encuestas estudiantiles anónimas, que son cruciales en los esfuerzos para conocer y entender las características del maltrato. Los estudios han demostrado que las encuestas anónimas, en general, son más eficaces en la adquisición de información sensible que las que no lo son ${ }^{(8)}$.

Este estudio presenta algunas limitaciones, siendo la percepción un hecho subjetivo, se convierte en una variable de difícil medición, que podría estar influenciada por diversos factores, como el sesgo de memoria, la inteligencia emocional del encuestado, la coyuntura del hecho, etc. por lo que la investigación de tipo cualitativa es la más apropiada. Sin embargo, la aplicación de una encuesta realizada sobre la base de estudios similares 
en población y utilizando procedimientos para su adecuación, permiten que el instrumento usado tenga una medición que se acerca a la realidad.

Concluimos que existe una alta prevalencia de maltrato hacia los estudiantes de Medicina en esta universidad pública del Perú, el tipo de maltrato más frecuente fue el psicológico, seguido del maltrato académico, físico y sexual. La percepción de maltrato se incrementa durante el ciclo de estudios en áreas clínico-quirúrgicas, donde los principales agresores los médicos docentes y residentes.

\section{REFERENCIAS BIBLIOGRÁFICAS}

1. Silver HK, Glicken AD. Medical student abuse. Incidence, severity and significance. JAMA. 1990;263(4):527-32. doi:10.1001/ jama.1990.03440040066030

2. Daugherty SR, Baldwin DC Jr, Rowley BD. Learning, satisfaction and mistreatment during medical internship: a national survey of working conditions. JAMA. 1998;279(15):1194-9. doi:10.1001/ jama.279.15.1194.

3. Cortés-Barré M. La letra con sangre entra: el maltrato en la formación médica. Univ Méd Bogotá (Colombia). 2010;51(1):43-8.

4. Fnais N, Soobiah C, Chen MH, Lillie E, Perrier L, Tashkhandi M, et al. Harassment and discrimination in medical training: a systematic review and meta-analysis. Acad Med. 2014;89(5):817-27. doi: 10.1097/ ACM.0000000000000200

5. Richman JA, Flaherty JA, Rospenda KM, Christensen ML. Mental health consequences and correlates of reported medical student abuse. JAMA. 1992;267(5):692-4. doi:10.1001/ jama.1992.03480050096032

6. Maida $S$ AM, Herskovic M V, Pereira $S$ A, Salinas-Fernández L, Esquivel C C. Percepción de conductas abusivas en estudiantes de Medicina. Rev Med Chil. 2006;134(12):1516-23. doi: 10.4067/ S0034-98872006001200004

7. Haviland MG, Yamagata $\mathrm{H}$, Werner LS, Zhang K, Dial TH, Sonne JL. Student mistreatment in medical school and planning a career in academic medicine. Teach Learn Med. 2011;23(3):231-7. doi: $10.1080 / 10401334.2011 .586914$.

8. Magnus R, Hawkins E, Miller M. Prevalence of harassment and discrimination among 1996 medical school graduates: a survey of eight US schools. JAMA. 1998;280(9):851-3.

9. Dent GA. Anonymous surveys to address mistreatment in medical education. Virtual Mentor. 2014;16(3):200-3. doi: 10.1001/ virtualmentor.2014.16.03.pfor1-1403.

10. Iglesias J, Saldivar D, Bermúdez V, Guzmán A. Maltrato del estudiante de Medicina. Percepción de 404 alumnos de cuarto, quinto y sexto años de la carrera. Med Univer. 2005;7(29):191-202.
11. Guevara-Cuéllar CA, Botero-Restrepo S, Borrero-Castro IL, Sinietra J. Prevalencia de abuso y factores asociados en una escuela de medicina colombiana. Colombia Med. 2011;42(4):490-500.

12. Maida $A M$, Vásquez $A$, Herskovic $V$, Calderón JL, Jacard M, Pereira A, et al. A report on student abuse during medical training. Med Teach. 2003;25(5):497-501. doi: $10.1080 / 01421590310001606317$

13. Owoaje ET, Uchendu OC, Ige OK. Experiences of mistreatment among medical students in a University in South West Nigeria. Niger J Clin Pract. 2012;15(2):2149. doi: 10.4103/1119-3077.97321.

14. Rautio A, Sunnari V, Nuutinen M, Laitala M. Mistreatment of university students most common during medical studies. BMC Med Educ. 2005;5:36. doi:10.1186/14726920-5-36

15. Carr PL, Ash AS, Friedman RH, Szalacha L, Barnett RC, Palepu A, et al. Faculty perceptions of gender discrimination and sexual harassment in academic medicine. Ann Intern Med. 2000;132(11):88996. doi:10.7326/0003-4819-132-11200006060-00007

16. Ulusoy H, Swigart V, Erdemir F. Think globally, act locally: understanding sexual harassment from a cross-cultural perspective. Med Edu. 2011;45(6):603-12. doi: 10.1111/j.1365-2923.2010.03918.x.

17. Moreno-Cubillos CL, Sepúlveda-Gallego LE. Discriminación y violencia contra los estudiantes de medicina de la Universidad de Caldas. Inv Ed Med. 2013;2(5):37-41.

18. Iftikhar R, Tawfiq R, Barabie S. Interns' perceived abuse during their undergraduate training at King Abdul Aziz University. Adv Med Educ Pract. 2014;5:159-66. doi: 10.2147/AMEP.S62890.

19. Consejo C, Viesca-Treviño C. Ética y relaciones de poder en la formación de médicos residentes e internos: Algunas reflexiones a la luz de Foucault y Bourdieu. Bol Mex His Fil Med. 2008;11(1):16-20.

20. Nagata-Kobayashi $S$, Sekimoto M, Koyama $\mathrm{H}$, Yamamoto W, Goto E, Fukushima O, et al. Medical student abuse during clinical clerkships in Japan. J Gen Intern Med.
2006;21(3):212-8. doi: 10.1111/j.15251497.2006.00320.x

21. Baldwin DC Jr, Daugherty SR, Eckenfels EJ. Student perceptions of mistreatment and harassment during medical school. A survey of ten United States schools. West J Med. 1991;155(2):140-5.

22. Ahmer S, Yousafzai AW, Bhutto N, Alam $S$, Sarangzai AK, Iqbal A. Bullying of Medical Students in Pakistan: a crosssectional questionnaire survey. PlosOne. 2008;3(12):e3889. doi: 10.1371/journal. pone.0003889.

23. Shoukat S, Anis M, Kella DK, Qazi F, Samad F, Mir F, et al. Prevalence of mistreatment or belittlement among medical students. A cross sectional survey at a private medical school in Karachi, Pakistan. Plos One. 2010;5(10):e13429. doi: 10.1371/journal.pone.0013429.

24. Cook AF, Arora VM, Rasinski KA, Curlin FA, Yoon JD. The prevalence of medical student mistreatment and its association with burnout. Acad Med. 2014;89(5):749-54. doi: 10.1097/ ACM.00000000000000204.

25. Richardson DA, Becker M, Frank RR, Sokol RJ. Assessing medical students' perceptions of mistreatment in their second and third years. Acad Med. 1997;72(8):728-30.

25. Organización Internacional de Trabajo. Directrices marco para afrontar la violencia laboral en el sector salud. $1^{a}$ ed. Ginebra: OIT; 2002.

26. Sheehan K, Sheehan DV, White $\mathrm{K}$, Leibowitz A, Baldwin DC Jr. A pilot study of medical student 'abuse'. Student perceptions of mistreatment and misconduct in medical school. JAMA. 1990;263(4):533-7.

Correspondencia: Fernando Munayco Guillén Dirección: Conjunto Habitacional Fonavi La Angostura W-12. Primera Etapa. Subtanjalla. Ica Teléfono: (511) 985558886 Correo electrónico: fernando.munayco. guillen@gmail.com 


\section{ANEXOS}

Anexo 1. Analisis de validez del constructo

\begin{tabular}{|c|c|c|c|c|}
\hline \multirow{2}{*}{ Matriz de componente rotado ${ }^{a}$} & \multicolumn{4}{|c|}{ Componente } \\
\hline & 1 & 2 & 3 & 4 \\
\hline ¿Te han gritado? & & 0,739 & 0,201 & \\
\hline ¿Has recibido comentarios negativos o despectivos? & & 0,707 & 0,200 & \\
\hline ¿Te han humillado? & 0,239 & 0,705 & & 0,243 \\
\hline ¿Te han insultado? & 0,291 & 0,693 & & 0,274 \\
\hline ¿Se han burlado respecto a tu género? & 0,238 & 0,245 & & 0,818 \\
\hline ¿Has recibido críticas injustificadas? & & 0,411 & & \\
\hline ¿Se han burlado respecto a tu étnia? & & 0,430 & & 0,576 \\
\hline ¿Has recibido amenazas verbales? & 0,265 & 0,716 & & \\
\hline ¿Te han golpeado? & 0,285 & 0,366 & 0,227 & 0,226 \\
\hline ¿Te han expuesto a trabajos de riesgo innecesario? & & 0,340 & & 0,625 \\
\hline ¿Te han asignado trabajos excesivos? & & 0,358 & & 0,664 \\
\hline ¿Te han asignado tareas como castigo? & & 0,217 & 0,605 & \\
\hline ¿Te han amenazado con jalarte en un curso? & & 0,511 & 0,401 & \\
\hline ¿Has tenido competencia desleal? & & & 0,582 & 0,232 \\
\hline ¿Otro a tomado el crédito de tu trabajo? & & & 0,653 & 0,195 \\
\hline ¿Me han discriminado por mi género? & 0,321 & & 0,373 & 0,652 \\
\hline ¿Le han hecho insinuaciones verbales sexuales o comentarios obscenos? & 0,579 & & 0,289 & 0,293 \\
\hline ¿Le han discriminado por su preferencia sexual? & 0,579 & & 0,216 & 0,381 \\
\hline Me han hecho propuestas indecentes & 0,826 & & & \\
\hline ¿Me han ostrado imagenes ofensivas de tipo sexual? & 0,739 & 0,252 & & \\
\hline ¿Le han hecho algún lenguaje corporal ofensivo de tipo sexual? & 0,723 & & 0,250 & \\
\hline ¿Le han tocado sin su consentimiento? & 0,805 & & & \\
\hline ¿Le han chantajeado sexualmente? & 0,756 & & 0,212 & \\
\hline
\end{tabular}

Método de extracción: análisis de componentes principales.

Método de rotación: Varimax con normalización Kaiser.

a. La rotación ha convergido en 7 iteraciones.

Anexo 2. Analisis factorial. Resumen del modelo

\begin{tabular}{cccc}
\hline \multirow{2}{*}{ Dimensión } & \multirow{2}{*}{ Alfa de Cronbach } & \multicolumn{2}{c}{ Varianza contabilizada para } \\
\cline { 2 - 4 } & & Total (autovalor) & Inercia \\
\hline 1 & 0,925 & 8,678 & 0,377 \\
2 & 0,876 & 6,174 & 0,268 \\
3 & 0,794 & 4,155 & 0,181 \\
4 & 0,774 & 3,850 & 0,167 \\
Total & & 22,857 & 0,994 \\
Media & $0,862^{\text {a }}$ & 5,714 & 0,248 \\
\hline
\end{tabular}

a. La media de alfa de Cronbach se basa en la media de auto valor.

Anexo 3. Análisis de confiabilidad

\begin{tabular}{lccc}
\hline & Ítems & Alfa de Crombach general & $\begin{array}{c}\text { Alfa de Crombach con elementos } \\
\text { estandarizados }\end{array}$ \\
\hline MALTRATO GENERAL & 23 & 0,862 & 0,890 \\
Componente sicológico & 8 & 0,762 & 0,828 \\
Componente físico & 3 & 0,599 & 0,609 \\
Componente académico & 4 & 0,635 & 0,647 \\
Componente sexual & 8 & 0,847 & 0,853 \\
\hline
\end{tabular}

